\title{
Toll-like receptor 4 agonist, lipopolysaccharide, increases the expression levels of cytokines and chemokines in human peripheral blood mononuclear cells
}

\author{
$\mathrm{DAN} \mathrm{PU}^{1}$ and WEI WANG ${ }^{2}$ \\ ${ }^{1}$ Clinical Skills Training Center, West China Hospital; ${ }^{2}$ Department of Pathology, \\ West China Second University Hospital, Sichuan University, Chengdu, Sichuan 610041, P.R. China
}

Received April 6, 2014; Accepted September 8, 2014

DOI: $10.3892 / \mathrm{etm} .2014 .2025$

\begin{abstract}
Toll-like receptors (TLRs) are members of the pattern recognition receptor family and are essential in the innate immune response. In total, 11 TLRs exist in humans, which are expressed in a variety of cells, including peripheral blood cells. TLR4 plays a significant role in the defense against gram-negative pathogens by recognizing the lipopolysaccharide (LPS) molecules in these bacteria. The aim of the present study was to detect the expression level variation of a number of major immune molecules in peripheral blood mononuclear cells (PBMCs) stimulated by LPS, in order to identify candidate genes involved in the biological functions mediated by TLR4. Reverse transcription quantitative polymerase chain reaction (RT-qPCR) analysis and an antibody chip were performed in the current study. The RT-qPCR results revealed a marked enhancement in the expression levels of various molecules, including cytokines, chemokines, growth factors and protein kinases. In addition, the antibody chip identified the increased secretion of crucial proinflammatory molecules in the supernatants collected from LPS-treated PBMCs. In conclusion, a large number of molecules were found to be involved in TLR4-mediated functions.
\end{abstract}

\section{Introduction}

Innate immunity is a major part of the immune system, playing a significant role in the acute inflammation induced by microbial infection or tissue damage (1). Innate immune cells, including monocytes, dendritic cells and T cells, can be recognized by their germ line-encoded pattern recognition receptors (PRRs), which are responsible for sensing the structure of microbial species (2). PRRs have also been shown to recognize endogenous ligands released from damaged cells and tissues (3).

Correspondence to: Dr Wei Wang, Department of Pathology, West China Second University Hospital, 20, Section 3, Renmin Nanlu, Sichuan University, Chengdu, Sichuan 610041, P.R. China

E-mail: bearwin2000@126.com

Key words: Toll-like receptor 4, lipopolysaccharide, peripheral blood mononuclear cells, cytokines, chemokines, kinases, antibody chip array
The Toll-like receptor (TLR) family is a well-characterized PRR family that is responsible for recognizing invading pathogens (4). TLR stimulation initiates a signal transduction pathway via the adaptor protein, MyD88, which induces the expression of proinflammatory cytokines, such as interleukin (IL)-6, IL-8 and tumor necrosis factor (TNF)- $\alpha$ (5). In total, 11 members constitute the human TLR family. Among them, TLR4 is a transmembrane protein specialized in the recognition of lipopolysaccharide (LPS), which is a component of gram-negative bacteria (6).

A previous study has demonstrated that the activation of TLR4 induces the expression of numerous proinflammatory molecules, which are essential in shaping the immune status of immune cells (7). However, the expression level variation of immune molecules within immune cells upon TLR4 stimulation remains unclear. In the current study, peripheral blood mononuclear cells (PBMCs) were isolated from healthy volunteers and stimulated by the TLR4 agonist, LPS, while the expression levels of various cytokines, chemokines, growth factors and kinases were screened. The aim of the present study was to confirm which types of immune molecules are involved in the activation of the TLR4 signaling pathway.

\section{Materials and methods}

Isolation and stimulation of PBMCs. Heparinized venous blood samples were isolated from three healthy male volunteers (aged 25-28 years) and PBMCs were separated by density separation over Ficoll-Hypaque. After washing twice with phosphate buffered-saline, the PBMCs were plated into 24-well plates with a total number of $2 \times 10^{6}$ cells/well. LPS was added to the PBMCs at a concentration of $100 \mathrm{ng} / \mathrm{ml}$. Supernatants were collected at $4 \mathrm{~h}$ following stimulation for use in the antibody chip. The present study was approved by the Ethics Committee of West China Hospital, Sichuan University (Sichuan, China) and written informed consent was obtained from the patients.

RNA extraction and cDNA synthesis. This procedure was performed as previously described (8). In brief, the total RNA from the PBMCs was extracted using a RNeasy mini kit (74104; Qiagen, Hilden, Germany) and quantified using 
Table I. Oligonucleotides used in quantitative polymerase chain reaction analysis.

\begin{tabular}{|c|c|c|c|}
\hline Gene & Forward primer & Reverse primer & GenBank number \\
\hline CCL5 & GACACCACACCCTGCTGCT & TACTCCTTGATGTGGGCACG & NM_002985 \\
\hline CCL7 & AGCAGAGGCTGGAGAGCTACA & GGGTCAGCACAGATCTCCTTGT & NM_006273 \\
\hline CCL8 & GTTTCTGCAGCGCTTCTGTG & TGGCTGAGCAAGTCCCTGA & Y10802 \\
\hline CCL15 & СCTCTCCTGCCTCATGCTTATT & CTCTGTCTCTGCATCATTTGTGAA & U58914 \\
\hline CCL17 & CCATCGTTTTTGTAACTGTGCAG & TGCATTCTTCACTCTCTTGTTGTTG & NM_002987 \\
\hline CCL22 & TGCGCGTGGTGAAACACT & GGTTAGCAACACCACGCCA & NM_002990 \\
\hline CCL24 & AGCCTTCTGTTCCTTGGTGTCT & GGGAGAGGGTATGACCACAGAG & NM_002991 \\
\hline CCL26 & CCAAGACCTGCTGCTTCCAA & GAATTCATAGCTTCGCACCCA & NM_006072 \\
\hline CCL28 & CTCGCCATCGTGGCCTT & GCAATGGGAAGTATGGCTTCTG & AF220210 \\
\hline c-Myc & CAAGACTCCAGCGCCTTCTC & GTTGAGTAACGAGCTGACCCC & AM393287 \\
\hline CTNNB & CATCGTGAGGGCTTACTGGC & GAGCAAGGCAACCATTTTCTG & XM_006712984 \\
\hline CXCL2 & AGGTGAAGTCCCCCGGAC & GCCCATTCTTGAGTGTGGCT & NM_002089 \\
\hline CXCL6 & GCTGAGAGTAAACCCCAAAACG & GGAGCACTGCGGGCC & NM_002993 \\
\hline GAPDH & GAAGGTGAAGGTCGGAGTC & GAAGATGGTGATGGGATTTC & J04038 \\
\hline IFN- $\beta$ & CAGCAATTTTCAGTGTCAGAAGCT & TCATCCTGTCCTTGAGGCAGT & M28622 \\
\hline IFN- $\gamma$ & CCAACGCAAAGCAATACATGA & CGCTTCCCTGTTTTAGCTGC & J00219 \\
\hline IL-1 $\beta$ & ACGAATCTCCGACCACCACT & CCATGGCCACAACAACTGAC & M15330 \\
\hline IL-2 & CAAGAATCCCAAACTCACCAGG & GACACTGAAGATGTTTCAGTTCTGT & J00264 \\
\hline IL-6 & GACCCAACCACAAATGCCA & GTCATGTCCTGCAGCCACTG & M14584 \\
\hline IL-7 & ACCAGTAGAAGACAATTGCATC & CCAGGTTTTCATCATCTTCAGCT & NM_001199888 \\
\hline IL-8 & CTGGCCGTGGCTCTCTTG & CCTTGGCAAAACTGCACCTT & NM_000584 \\
\hline IL-12 & CGGTCATCTGCCGCAAA & CAAGATGAGCTATAGTAGCGGTCCT & M65272 \\
\hline IL-15 & GACCCCACCAAAGCTGGAC & TCACAGTGCTGCTGTCTGCTG & M90391 \\
\hline IL-23 & GAAGGCTCCGCTCTGCAAT & TCTGGGTCTTCTCGATGGCA & L06801 \\
\hline IP-10 & TGAAATTATTCCTGCAAGCCAA & CAGACATCTCTTCTCAСССТTCTTT & NM_001565 \\
\hline JNK & GCTAATTCTGTACCAATGTC & GAAGAGTGCACGTCAGGAAC & NM_139049 \\
\hline MAP3K & CCTGCTCGGTGCACGATGCTG & СTCTGTCTCTTCACGTGGCGG & NM_003954 \\
\hline MAP3K1 & CTTTTAAGTCAGAAGTTGCTG & СТTСТССАТТTТСААССТGC & AF042838 \\
\hline MIP-3 $\alpha$ & TCCTGGCTGCTTTGATGTCA & TCAAAGTTGCTTGCTGCTTCTG & NM_004591 \\
\hline MIP-3 $\beta$ & GGCACCAATGATGCTGAAGA & GAAGTTCCTCACGATGTACCCAG & NM_006274 \\
\hline $\mathrm{NF}-\kappa \mathrm{B}$ & AGAGTGCTGGAGTTCAGGATA & AAGGTGGATGATTGCTAAGTGT & AJ 271718 \\
\hline PTEN & ACCATAACCCACCACAGC & CAGTTCGTCCCTTTCCAG & NM_058074 \\
\hline PI3K & CCTGGGGGTTGGTGGCTGTTC & GTCTGGCTGGAATGATGCTATC & NM_006219 \\
\hline STAT3 & CCTACAAAGGGGACCCCATTGTAC & CAGGGAATTTGACCAGCAACC & NM_213662 \\
\hline TGF- $\alpha$ & TATCGACATGGAGCTGGTGAAG & CAGCTTGGACAGGATCTGGC & X02812 \\
\hline TNF- $\beta$ & GGTGCTTGTTCCTCAGCCTC & CAGGCAGAAGAGCGTGGTG & M10988 \\
\hline VEGF & GACTTGAGTTGGGAGGGGAA & GAGGCTCAGCGCCAGGGCTGGG & AF024710 \\
\hline
\end{tabular}

CCL, chemokine (C-C motif) ligand; CTNNB, cadherin-associated protein $\beta$; CXCL, chemokine (C-X-C motif) ligand; GAPDH, glyceraldehyde 3-phosphate dehydrogenase; IFN, interferon; IL, interleukin; IP, interferon $\gamma$-induced protein; JNK, c-Jun N-terminal kinase; MAP3K, mitogen-activated protein kinase kinase kinase; MIP, macrophage inflammatory protein; NF, nuclear factor; PTEN, phosphatase and tensin homolog; PI3K, phosphatidylinositol-4,5-bisphosphate 3-kinase; STAT, signal transducer and activator of transcription; TGF, transforming growth factor; TNF, tumor necrosis factor; VEGF, vascular endothelial growth factor.

a NanoDrop 3300 Fluorospectrometer (Thermo Fisher Scientific, Wilmington, DE, USA). cDNA was synthesized using a ReverTra Ace qPCR kit (FSQ-101; Toyobo, Kagoshima, Japan) and the reverse transcription (RT) conditions were as follows: $65^{\circ} \mathrm{C}$ for $5 \mathrm{~min}, 37^{\circ} \mathrm{C}$ for $15 \mathrm{~min}$ and $98^{\circ} \mathrm{C}$ for $5 \mathrm{~min}$.

Quantification polymerase chain reaction $(P C R)$. This procedure was performed as previously described (8). In brief, quantitative PCR (qPCR) was performed to amplify the synthesized cDNA using the RealMaster Mix Reagent (SYBR Green; FP202; Tiangen Biotech Co., Ltd., Beijing, China). An iCycler iQTM Optical Module (Beckman Coulter, Fullerton, CA, USA) was used for RT-qPCR under the following conditions: One cycle at $95^{\circ} \mathrm{C}$ for $30 \mathrm{sec}, 40$ cycles at $95^{\circ} \mathrm{C}$ for $30 \mathrm{sec}$, $58^{\circ} \mathrm{C}$ for $30 \mathrm{sec}$ and $72^{\circ} \mathrm{C}$ for $30 \mathrm{sec}$, followed by a melt curve between 55 and $95^{\circ} \mathrm{C}$ in $0.5^{\circ} \mathrm{C}$-increments and 10 -sec intervals. 
A

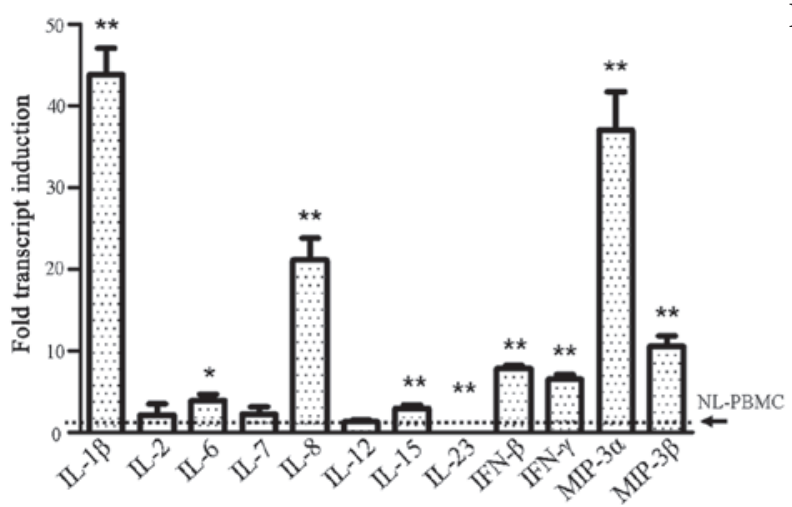

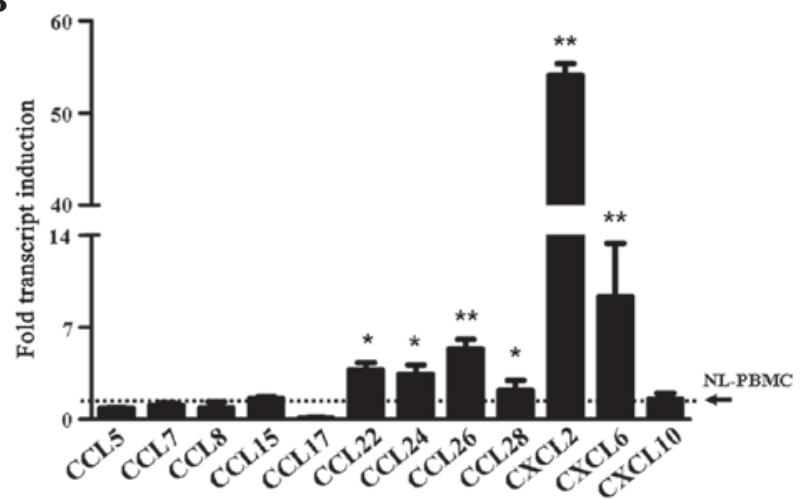

Figure 1. Gene expression level variation in (A) cytokines and (B) chemokines, as detected by quantitative reverse transcription polymerase chain reaction. ${ }^{*} \mathrm{P}<0.05$ and ${ }^{* *} \mathrm{P}<0.001$, vs. control group. IL, interleukin; IFN, interferon; MIP, macrophage inflammatory protein; CCL, chemokine (C-C motif) ligand; CXCL, chemokine (C-X-C motif) ligand; NL-PBMC, normal peripheral blood mononuclear cell.
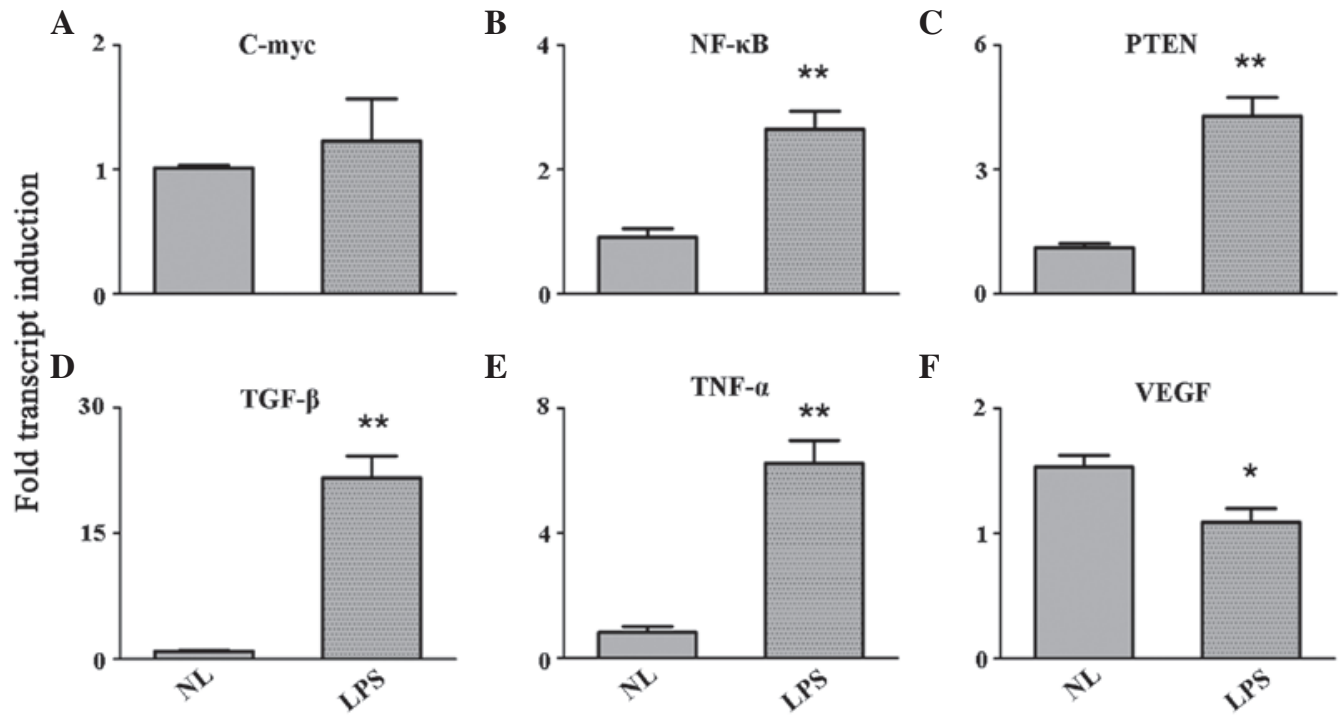

$\mathbf{F}$

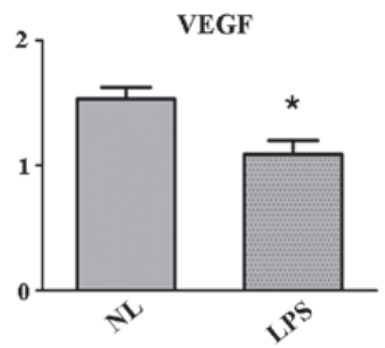

Figure 2. Expression level variation of the growth factors, (A) c-Myc, (B) NF- $\kappa$ B, (C) PTEN, (D) TGF- $\beta$, (E) TNF- $\alpha$ and (F) VEGF, as detected by quantitative reverse transcription polymerase chain reaction. ${ }^{*} \mathrm{P}<0.05$ and ${ }^{* *} \mathrm{P}<0.001$, vs. control group. NF, nuclear factor; PTEN, phosphatase and tensin homolog; TGF, transforming growth factor; TNF, tumor necrosis factor; VEGF, vascular endothelial growth factor; NL, normal PBMCs; LPS, lipopolysaccharide-treated PBMCs; PBMCs, peripheral blood mononuclear cells.

All the tests were performed in triplicate and the primers used are shown in Table I.

Antibody chip. The supernatants collected from the TLR4-stimulated and unstimulated PBMCs were arrayed for molecule secretion using the RayBio ${ }^{\circledR}$ Human Antibody Array C-Series 1000 (RayBiotech, Inc., Norcross, GA, USA), according to the manufacturer's instructions. Blots were analyzed using ImageJ software (National Institutes of Health, Bethesda, MD, USA).

Statistical analysis. Data analysis was performed using the Bio-Rad iQ5 software (Bio-Rad Laboratories, Hercules, CA, USA), with glyceraldehyde 3-phosphate dehydrogenase as the internal control and normal PBMCs as the negative control. The results are expressed as the mean \pm standard error of the mean and were analyzed using SPSS 16.0 software (SPSS, Inc., Chicago, IL, USA). $\mathrm{P}<0.05$ and $\mathrm{P}<0.001$ were considered to indicate a statistically significant difference when compared with the control group. The figures were obtained using GraphPad Prism 5 software (GraphPad Software, Inc., La Jolla, CA, USA).

\section{Results}

TLR4 agonist increases the expression levels of cytokines and chemokines. RT-qPCR was performed to screen the expression levels of a number of cytokines and chemokines, in order to identify candidate genes responsible for the LPS-mediated changes in PBMCs. The expression level variation of a number of these genes may significantly affect the biological function of the PBMCs, induced by microenvironment or pathogen stimulation.

With regard to cytokine detection, the TLR4 agonist, LPS, was found to significantly increase the expression levels of several major cytokines $(\mathrm{P}<0.001)$, including IL-1 $\beta$, IL-8, IL-15, interferon (IFN) $-\beta$, IFN- $\gamma$, macrophage inflammatory protein (MIP)-3 $\alpha$ and MIP- $\beta$. In addition, LPS slightly increased the expression level of IL-6 $(\mathrm{P}<0.05)$. IL-23 was 
A

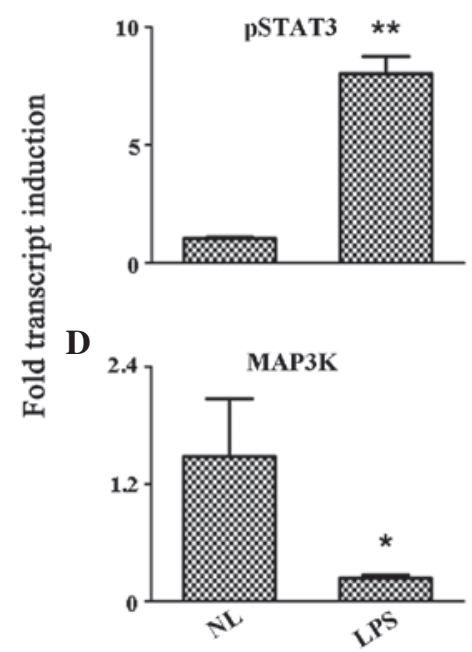

B
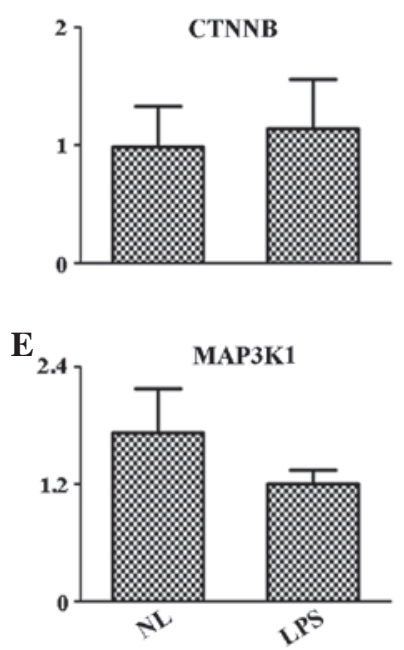

C
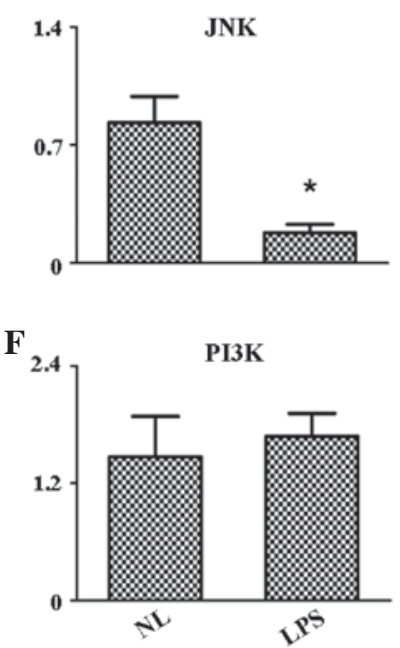

Figure 3. Expression level variation of the protein kinases, (A) pSTAT3, (B) CTNNB, (C) JNK, (D) MAP3K, (E) MAP3K1 and (F) P13K, as detected by quantitative reverse transcription polymerase chain reaction. ${ }^{*} \mathrm{P}<0.05$ and ${ }^{* *} \mathrm{P}<0.001$, vs. control group. pSTAT3, phosphorylated signal transducer and activator of transcription 3; CTNNB, cadherin-associated protein $\beta$; JNK, c-Jun N-terminal kinase; MAP3K, mitogen-activated protein kinase kinase kinase; PI3K, phosphatidylinositol-4,5-bisphosphate 3-kinase; NL, normal PBMCs; LPS, lipopolysaccharide-treated PBMCs; PBMCs, peripheral blood mononuclear cells.
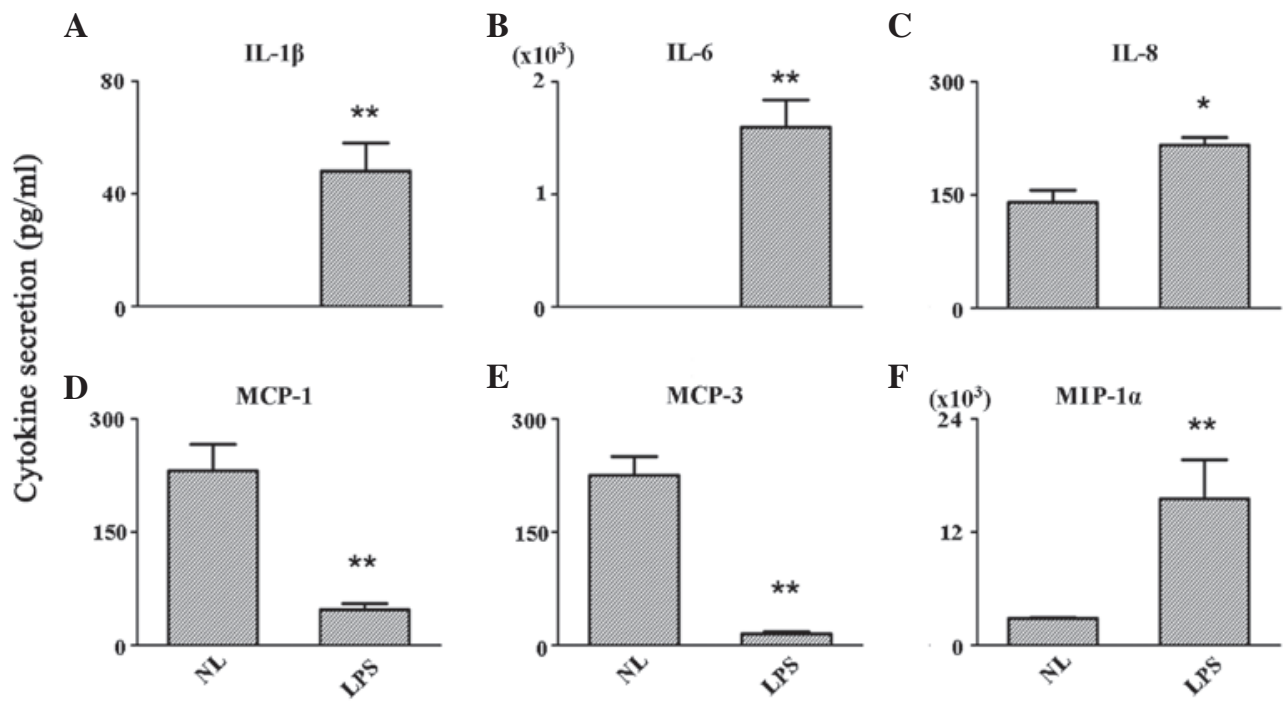

Figure 4. Secretion levels of (A) IL-1 $\beta$, (B) IL-6, (C) IL-8, (D) MCP-1, (E) MCP-3 and (F) MIP-1 $\alpha$ in the supernatant. *P<0.05 and ** $<<0.001$, vs. control group. IL, interleukin; MCP, monocyte chemoattractant protein; MIP, macrophage inflammatory protein; NL, normal control PBMCs; LPS, lipopolysaccharide-treated PBMCs; PBMCs, peripheral blood mononuclear cells.

the only downregulated cytokine detected ( $\mathrm{P}<0.001$; Fig. 1A). During the chemokine assay, only the chemokine (C-C motif) ligand (CCL) 26, chemokine (C-X-C motif) ligand (CXCL) 2 and CXCL6 were found to be evidently enhanced upon LPS stimulation $(\mathrm{P}<0.001)$, although CCL22, CCL24 and CCL28 were also found to be significantly different compared with the controls $(\mathrm{P}<0.05$; Fig. 1B).

LPS stimulation enhances the expression levels of growth factors. Growth factors were screened to obtain their expression levels following LPS stimulation. The results indicated an evident upregulation of nuclear factor $(\mathrm{NF})-\kappa \mathrm{B}$, phosphatase and tensin homolog (PTEN), transforming growth factor (TGF)- $\beta$ and TNF- $\alpha$ in the PBMCs activated by the TLR ligand
$(\mathrm{P}<0.001)$. The expression of $\mathrm{c}-\mathrm{Myc}$ remained unchanged, while the expression of vascular endothelial growth factor (VEGF) was inhibited ( $\mathrm{P}<0.05$; Fig. 2).

Activation of the TLR4 signaling pathway has no evident effect on the expression levels of kinases. TLR4 agonist stimulation performed on PBMCs may induce the activation of protein kinases. Therefore, a number of protein kinase signaling pathways were analyzed in the study. The results indicated that the expression of phosphorylated signal transducer and activator of transcription 3 (pSTAT3) was significantly enhanced in PBMCs following LPS stimulation $(\mathrm{P}<0.001)$. However, cadherin-associated protein $\beta$ (CTNNB), mitogen-activated protein kinase kinase kinase 1 (MAP3K1) 
and phosphatidylinositol-4,5-bisphosphate 3-kinase (PI3K) expression levels remained unchanged during the detection. Notably, the expression levels of two important kinases, c-Jun N-terminal kinase (JNK) and MAP3K, were inhibited following LPS treatment $(\mathrm{P}<0.05$; Fig. 3).

LPS induces the secretion of proinflammatory molecules. Supernatants collected from the LPS-treated and untreated PBMCs were analyzed to determine the secretion levels of major immune molecules using an antibody chip. A total of 20 proteins, including $\alpha$-fetoprotein, albumin, E-selectin, intercellular adhesion molecule-1, IFN- $\alpha$, IFN- $\gamma$, IL-10, IL-12, IL-18, IL-1 $\beta$, IL-4, IL-5, IL-6, IL-8, monocyte chemoattractant protein (MCP)-1, MCP-3, MIP-1 $\alpha$, Notch-1, TGF- $\beta$ and VEGF, were selected for analysis. The results revealed that only six of the aforementioned proteins were affected by TLR4 activation (Fig 4). Among these, the secretion levels of IL-1 $\beta$, IL-6 and MIP- $1 \alpha$ were significantly enhanced $(\mathrm{P}<0.001)$, while IL-8 secretion was moderately increased $(\mathrm{P}<0.05)$. In addition, the secretion levels of MCP-1 and MCP-3 were inhibited.

\section{Discussion}

Vertebrates have evolved systems of immune defense to eliminate invading pathogens. The innate immune system is the first line of the host defense against microorganisms and recognizes the conserved components of pathogens via pattern recognition receptors (PRRs) (9). Among the 11 human TLRs, TLR3, TLR7, TLR8 and TLR9 are expressed on the surface of endosomes, while TLR1, TLR2, TLR4, TLR5, TLR6, TLR10 and TLR11 are located on the cellular surface (10). TLR4 plays an important role in the protection against fungi by recognizing LPS components. Stimulation with a TLR4 agonist induces proinflammatory signals, as well as the production of type I IFNs (11).

In the present study, PBMCs were isolated from healthy volunteers and stimulated by the TLR 4 agonist, LPS. At $4 \mathrm{~h}$ after the treatment, the supernatant and cellular RNA were isolated and assayed using an antibody chip and RT-qPCR, respectively. Using these methods, the immune molecules involved in the immunological signature of PBMCs can be identified and the TLR4 signaling pathway can be further understood. The molecules selected in the present study exhibit a number of important biological functions in vivo. In addition to increasing the expression levels of IL-8 and IL-6 (12), TLR4 agonist stimulation was found to influence numerous immunomodulatory factors. Among the cytokines examined, the expression levels of IL-1 $\beta$, IL-6, IL-8, IL-15, IFN- $\beta$, IFN- $\gamma$, MIP-3 $\alpha$ and MIP-3 $\beta$ increased markedly. During chemokine detection, the expression levels of CCL22, CCL24, CCL26, CXCL2 and CXCL6 were upregulated upon LPS stimulation. In addition, the expression levels of several growth factors, including NF- $\kappa$ B, PTEN, TNF- $\alpha$ and TGF- $\beta$, were enhanced. Detecting the expression levels of protein kinases, which play important roles in TLR-mediated biological functions, was important in this study. The expression level of pSTAT3 was found to increase in LPS-treated PBMCs, while the expression levels of CTNNB, MAP3K1 and PI3K remained unchanged in the treated and untreated groups. However, the expression of JNK was inhibited following LPS treatment. These results indicated that the activation of TLR4 had varying effects on the different kinase pathways. The cytokine levels were detected in the supernatants of LPS-treated PBMCs, and the secretion levels of IL-1 $\beta$, IL-6, IL-8 and MIP- $1 \alpha$ were found to be enhanced, indicating the important role of TLR4 in shaping the immune status of PBMCs.

Compared with previous studies, the present study analyzed a greater number of immune molecules that may be important in TLR-related functions. However, a limitation of the study was that the variation in the expression levels of immune molecules was only detected at a gene level. Future studies should analyze the association between these candidate immune molecules and TLR-mediated functions and should investigate the MEprotein expression levels.

\section{Acknowledgements}

This study was supported by a grant from the National Natural Science Foundation of China (no. 81101261).

\section{References}

1. Takeuchi $\mathrm{O}$ and Akira S: Pattern recognition receptors and inflammation. Cell 140: 805-820, 2010.

2. Hou B, Reizis B and DeFranco AL: Toll-like receptors activate innate and adaptive immunity by using dendritic cell-intrinsic and -extrinsic mechanisms. Immunity 29: 272-282, 2008.

3. Miyake K: Innate immune sensing of pathogens and danger signals by cell surface Toll-like receptors. Semin Immunol 19: 3-10, 2007.

4. Blasius AL and Beutler B: Intracellular Toll-like receptors. Immunity 32: 305-315, 2010.

5. Yamamato M, Sato S, Mori K, Hoshino K, Takeuchi O, Takeda K and Akira S: Cutting edge: a novel Toll/IL-1 receptor domain-containing adapter that preferentially activates the IFN-beta promoter in the Toll-like receptor signaling. J Immunol 169: 6668-6672, 2002.

6. Ghosh TK, Mickelson DJ, Solberg JC, Lipson KE, Inglefield JR and Alkan SS: TLR-TLR cross talk in human PBMC resulting in synergistic and antagonistic regulation of type- 1 and 2 interferons, IL-12 and TNF-alpha. Int Immunopharmacol 7: 1111-1121, 2007.

7. Kaisho T, Takeuchi O, Kawai T, Hoshino K and Akira S: Endotoxin-induced maturation of MyD88-deficient dendritic cells. J Immunol 166: 5688-5694, 2001.

8. Activation of the TLR1/2 pathway induces the shaping of the immune response status of peripheral blood leukocytes. Exp Ther Med 7: 1708 1712, 2014

9. Beutler B: Inferences, questions and possibilities in Toll-like receptor signalling. Nature 430: 257-263, 2004.

10. Kopp $E$ and Medzhitov R: Recognition of microbial infection by Toll-like receptors. Curr Opin Immunol 15: 396-401, 2003.

11. Shan JY, Ji WZ, Li HT, Tuxun T, Lin RY and Wen H: TLR2 and TLR4 expression in peripheral blood mononuclear cells of patients with chronic cystic echinococcosis and its relationship with IL-10. Parasite Immunol 33: 692-696, 2011.

12. Kaisho T and Akira S: Toll-like receptor function and signaling. J Allergy Clin Immunol 117: 979-987, 2006. 